\title{
The contribution of different risk factors in the occurrence of stroke
}

\author{
Precious Barnes ${ }^{1}$, Kwabena Nsiah ${ }^{2}$, Faustina Mensah ${ }^{2}$ \\ ${ }^{1}$ Department of Biochemistry, University of Cape Coast, Cape Coast, Ghana \\ ${ }^{2}$ Department of Biochemistry and Biotechnology, Kwame Nkrumah University of Science and Technology, Kumasi, Ghana
}

Email address:

p.barnes@uccsms.edu.gh (P. Barnes), precious4bill@yahoo.com (P. Barnes)

\section{To cite this article:}

Precious Barnes, Kwabena Nsiah, Faustina Mensah. The Contribution of Different Risk Factors in the Occurrence of Stroke. American Journal of Health Research. Vol. 2, No. 6, 2014, pp. 387-391. doi: 10.11648/j.ajhr.20140206.21

\begin{abstract}
Background: Although stroke cause substantial morbidity and mortality, it is unclear how individuals are affected, or a group of risk factors contribute to stroke outcomes especially in developing countries. Methods: This study evaluated the risk factors of stroke. This was a cross-sectional study of stroke patients from Komfo Anokye Teaching Hospital; Adult enrolees who were eligible for inclusion were persons who have had stroke within twenty-four hours of onset without any medication. Questionnaires were used to gather information on medical history, demographic features and lifestyle. The lipid profile, fasting blood sugar and blood pressure were measured, likewise the determination of aminotransferases and gammaglutamyltransferase. Results: Out of 224 stroke patients, of mean age $65.64 \pm 1.08$ SEM years, used for the study, $56.7 \%$ of the patients had hyperglycaemia, whereas $47.2 \%$ were known diabetics. Hypertriglyceridaemiawas found in $55.8 \%$, followed by $30.8 \%$ of the patients who had a reduced HDL-C and $17.95 \%$ of them with increased LDL-C. About $20 \%$ of the patients had raised AST, 7.1\% raised ALT and 14.7\% raised GGT above the normal levels. As high as $63 \%$ of stroke patients had high blood pressure. Conclusions: There were a high prevalence of both hypertension and hyperglycaemia among the stroke patients. Based on this study, it is important to stress the need for educating the stroke patients and/or their caregivers, on the aetiology of stroke and the treatment options. The general population should also be educated on primary preventive measures for all age groups and both genders.
\end{abstract}

Keywords: Stroke, Risk Factors, Dyslipidimea, Hyperglycaemia

\section{Introduction}

Stroke is becoming a common clinical condition even in developing countries. The probability of stroke occurrence rises with the number and severity of vascular risk factors (Algra, 2006). An emerging entity that incorporates risk stratification with the goal of mitigating vascular risk is the stroke (McNeill et al., 2006). The risk factors of stroke are highly prevalent worldwide, and several studies have suggested that individuals with the risk factors are at high risk for experiencing first and recurrent stroke. The prevalence of the risk factors are expected to substantially increase in the future, alongside the growing obesity epidemic (Ogden and Algra, 2006), a rise that will likely be associated with an even heavier burden of stroke on the society. It is important to identify persons with risk factors in order to initiate lifestyle modification and the use of drugs treatment to prevent cardiovascular disease and stroke
(Grundy et al., 2004).

Due to the paucity of information on risk factors to stroke and the accompanying biochemical changes in Africa, particularly, this study was undertaken to investigate the risk factors present in stroke.

\section{Methodology}

\subsection{Selection of Patients}

Stroke in-patients were selected from the Komfo Anokye Teaching Hospital (KATH) in Kumasi, from specific wards for male and female stroke patients. There were 117 males and 107 females within an age range of 25-120 years. The inclusion criteria for the selection were patients who have had a stroke in less than twenty-four hours without any medication. The patients were those admitted with sudden 
onset of characteristic neurological deficit and having distinctive neurological signs. Patients with loss of consciousness due to head injury, coma, trauma, tumour, poisoning or epilepsy were not included. This was a crosssectional study. A questionnaire was administrated to each patient's caregiver in order to obtain the following information; demographic features (age, sex), socioeconomic status (education and employment), medical history with specific attention to hypertension and diabetes and lifestyle (smoking and drinking). The study started from October 2009 and ended in January 2011. The Committee for Human Research Publications and Ethics of the Komfo Anokye Teaching Hospital gave the approval for the study.

\subsubsection{Specimen Collection and Processing}

About $5 \mathrm{ml}$ of venous blood specimens were collected into non- anticoagulated tubes and were labeled with patients identifications. Another $5 \mathrm{ml}$ of their blood were also collected into ethylenediaminetetra acetic acid (EDTA) tubes. The blood specimens in both tubes were centrifuged to obtain serum and plasma, respectively. The samples were centrifuged in a speed of 12000-13000 rpm at 5 minutes.

\subsubsection{Fasting blood sugar}

The test was done with Biotechnical Instrument (BT-3000 PLUS; made in Vila do Conde, Portugal) an automated analyzer.

\subsubsection{Liver Function Tests}

Fifty microlitres $(50 \mu \mathrm{l})$ of serum samples were put into sample tubes which were labeled with the patients identifications. The samples were uploaded onto the sample plate of the analyzer. A command "run" was issued and results were printed out after 30 minutes

\subsection{Blood Pressure}

Blood pressure was taken using a mercury sphygmomanometer and stethoscope. Measurements were taken from the left upper arm after patients had been sitting for 5 minute in accordance with recommendation of American Heart Association (Booth, 1999).

\subsection{Statistical Analysis}

Statistical analysis was conducted by calculating simple means and standard error of mean for continuous variables, and frequency counts and percentages for categorical variables. Fischer's, test, Pearson's correlation and student's t-test analyses were carried out on variables. Significance was set at $\mathrm{p}<0.05$.

\section{Results}

\subsection{Characteristics of Stroke Patients}

From Table 1, the mean age of all the stroke patients was $65.64 \pm 1.08$ SEM years. The mean systolic and diastolic blood pressures for the patients were $179.80 \pm 3.47$ and $107.80 \pm 2.33 \mathrm{mmHg}$ respectively. Whereas about $15 \%$ of the patients smoked cigarette, about $30 \%$ of the patients consumed alcoholic beverages. Over half of the patients were known hypertensive patients (i.e. $51.3 \%$ ), about $32 \%$, known diabetics. Seven of the stroke patients died, which constitute $3 \%$ of the stroke patients during the course of the study.

Table 1. General characteristics of the study population stratified by gender.

\begin{tabular}{|c|c|c|c|c|}
\hline Variable & Total $(n=224)$ & Female $(n=107)$ & Male $(n=117)$ & P value \\
\hline \multicolumn{5}{|c|}{ Socio-demographic data } \\
\hline Age (years) & $65.64 \pm 1.08$ & $67.51 \pm 1.74$ & $63.92 \pm 1.31$ & 0.0965 \\
\hline SBP (mmHg) & $179.80 \pm 3.47$ & $180.60 \pm 4.93$ & $179.10 \pm 4.91$ & 0.8293 \\
\hline Smoking & $33(14.7 \%)$ & $2(1.9 \%)$ & $31(26.5 \%)$ & $<0.0001$ \\
\hline Alcohol & $68(30.4 \%)$ & $13(12.1 \%)$ & $55(47.0 \%)$ & $<0.0001$ \\
\hline \multicolumn{5}{|l|}{ Lipid profile } \\
\hline HDL-C (mmol/l) & $1.77 \pm 0.13$ & $1.92 \pm 0.23$ & $1.64 \pm 0.14$ & 0.2875 \\
\hline $\mathrm{TG}(\mathrm{mmol} / \mathrm{l})$ & $2.52 \pm 0.14$ & $2.25 \pm 0.17$ & $2.83 \pm 0.23$ & 0.0426 \\
\hline $\mathrm{TC}(\mathrm{mmol} / \mathrm{l})$ & $3.81 \pm 0.18$ & $3.54 \pm 0.26$ & $4.06 \pm 0.25$ & 0.1523 \\
\hline \multicolumn{5}{|l|}{ Liver function test } \\
\hline $\operatorname{AST}(\mathrm{U} / \mathrm{L})$ & $30.02 \pm 0.99$ & $29.34 \pm 1.33$ & $31.08 \pm 1.45$ & 0.3778 \\
\hline $\operatorname{ALT}(\mathrm{U} / \mathrm{L})$ & $26.85 \pm 1.04$ & $24.82 \pm 1.03$ & $27.52 \pm 1.30$ & 0.1113 \\
\hline GGT (U/L) & $35.54 \pm 1.49$ & $34.13 \pm 1.71$ & $35.14 \pm 1.71$ & 0.6782 \\
\hline FBS (mmol/l) & $8.21 \pm 0.30$ & $7.58 \pm 0.40$ & $8.79 \pm 0.45$ & 0.0454 \\
\hline Known hypertensive & $115(51.3 \%)$ & $50(46.7 \%)$ & $65(55.6 \%)$ & 0.2285 \\
\hline Known diabetes & $71(31.7 \%)$ & $30(28.0 \%)$ & $41(35.0 \%)$ & 0.3145 \\
\hline Death & $7(3.1 \%)$ & $6(5.6 \%)$ & $1(0.9 \%)$ & 0.0562 \\
\hline
\end{tabular}

Continuous data are presented as mean \pm SEM and categorical data presented as proportion. The continuous data were compared using unpaired t-test whilst categorical data were compared using Fischer's exact test.

Meanings of abbreviations;Gamma-glutamyltransferase (GGT), Aspartate aminotransferase (AST), Alanine aminotransferase (ALT),Systolic Blood Pressure (SBP), Diastolic Blood Pressure (DBP), Total Cholesterol (TC), Triglyceride (TG),High Density Lipoprotien-cholesterol (HDL-C),Low Density Lipoprotiencholesterol (LDL-C),Fasting Blood Sugar (FBS). 
The mean age, as well as the mean systolic and diastolic blood pressure of the female participants was similar to that of their male counterparts. The proportions of the male participants who smoked cigarettes $(26.5 \%)$ and consumed alcoholic beverages (47.0\%) were significantly higher, as compared to the females who smoked $(1.9 \%)$ and consumed alcoholic beverages (12.1\%) as shown in Table 1.

Except for the mean triglyceride level that was significantly $(\mathrm{p}=0.0426)$ higher among the male participants $\left(2.83 \pm 0.23 \mathrm{mmol} \mathrm{l}^{-1}\right)$ as compared to the female subjects $\left(2.25 \pm 0.17 \mathrm{mmol} \mathrm{l}^{-1}\right)$, all the other lipid profile parameters were similar in both genders. The mean levels of the markers of liver dysfunction were also similar in both genders. Fasting blood glucose level was significantly higher ( $\mathrm{p}=$ $0.0454)$ in the male subjects $\left(8.79 \pm 0.45 \mathrm{mmol} \mathrm{l}^{-1}\right)$, as compared to their female counterparts $\left(7.58 \pm 0.40 \mathrm{mmol} \mathrm{l}^{-1}\right)$ as shown in Table 1.

The highest prevalence of lipid disorder was seen in hypertriglyceridaemia $(55.8 \%)$, followed by reduced HDLcholesterol (30.8\%), hypercholesterolaemia (23.2\%) and increased LDL cholesterol level (17.9\%) as shown in Table 2.
When the studied population was stratified based on gender, there was no significant difference in the percentages of the various dyslipidaemia parameters, and the orders of dyslipidaemia were similar to that of the total studied population.

Using the blood pressure grading system (World Health Organisation/ International Society of Hypertension, 1999), about $63 \%$ of the patients met the severe hypertension criterion, followed by mild, moderate and high normal blood pressure. When the classification was done by gender, there were no significant differences in the level of the various grading (Table 2).Whereas about $20 \%$ of the entire patients' population had their AST level raised, 7.1\% had their ALT level raised and $14.7 \%$ had their GGT level raised above the normal level (Table 2).

More than half of the patients had hyperglycaemia (56.7\%), impaired fasting glucose was seen in about $10 \%$ and diabetes was found in about $47 \%$ of the patients. Significant proportion of the male subjects had hyperglycaemia $(65.0 \%)$ and diabetes $(53.8 \%)$ as compared to their female counterparts, in whom $47.7 \%$ were hyperglycaemia and $40.2 \%$ were diabetic.

Table 2.Prevalence of dyslipidaemia, hypertension, and abnormal liver function test and glycaemic control disorders.

\begin{tabular}{|c|c|c|c|c|c|c|}
\hline Variables & \multicolumn{2}{|c|}{ Cut off or ranges } & Total $n=224$ & Female $n=107$ & Male n=117 & P value \\
\hline \multicolumn{7}{|l|}{ Dyslipidaemia } \\
\hline Hypercholesterolaemia & \multicolumn{2}{|c|}{$\mathrm{TC}\left(>5.2 \mathrm{mmol} \mathrm{L}^{-1}\right)$} & $52 / 224(23.2 \%)$ & $21 / 107(19.6 \%)$ & $31 / 117(26.5 \%)$ & 0.2681 \\
\hline Hypertriglyceridaemia & \multicolumn{2}{|c|}{$\mathrm{TG}\left(>1.8 \mathrm{mmol} \mathrm{L}^{-1}\right)$} & $125 / 224(55.8 \%)$ & $58 / 107(54.2 \%)$ & $67 / 117(57.3 \%)$ & 0.6872 \\
\hline Reduced HDL cholesterol & \multicolumn{2}{|c|}{ HDL-C $\left(<1.0 \mathrm{mmol} \mathrm{L}^{-1}\right)$} & $69 / 224(30.8 \%)$ & $32 / 107(29.9 \%)$ & $37 / 117(31.6 \%)$ & 0.8849 \\
\hline Increased LDL cholesterol & \multicolumn{2}{|c|}{ LDL-C $\left(>4.1 \mathrm{mmol} \mathrm{L}^{-1}\right)$} & $40 / 224(17.9 \%)$ & $22 / 107(20.6 \%)$ & $18 / 117(15.4 \%)$ & 0.3830 \\
\hline \multicolumn{7}{|l|}{ Blood pressure disorder } \\
\hline Blood pressure category & Systolic & Diastolic & & & & \\
\hline Optimal & $<120 \mathrm{mmHg}$ & $<80 \mathrm{mmHg}$ & $13 / 18(72.2 \%)$ & $5 / 7(71.4 \%)$ & $8 / 11(72.7 \%)$ & 1.0000 \\
\hline Normal & $120-129$ & $80-84$ & $3 / 12(25.0 \%)$ & $1 / 7(14.3 \%)$ & $2 / 5(40.0 \%)$ & 0.5227 \\
\hline High normal & $130-139$ & $85-89$ & $1 / 21(4.8 \%)$ & $1 / 12(8.3 \%)$ & $0 / 9(0.0 \%)$ & 1.0000 \\
\hline Mild & $140-159$ & $90-99$ & $16 / 29(55.2 \%)$ & $7 / 14(50.0 \%)$ & $9 / 15(60.0 \%)$ & 0.7152 \\
\hline Moderate & $160-179$ & $100-109$ & $10 / 43(23.3 \%)$ & $2 / 16(12.5 \%)$ & $8 / 27(29.6 \%)$ & 0.2757 \\
\hline Severe & $\geq 180$ & $\geq 110$ & $64 / 101(63.4 \%)$ & $32 / 51(62.7 \%)$ & $32 / 50(64.0 \%)$ & 1.0000 \\
\hline \multicolumn{7}{|l|}{ Liver dysfunction } \\
\hline $\operatorname{AST}\left(\mathrm{U} \mathrm{L}^{-1}\right)$ & \multicolumn{2}{|l|}{$>40$} & $44 / 224(19.6 \%)$ & $17 / 107(15.9 \%)$ & $27 / 117(23.1 \%)$ & 0.1835 \\
\hline $\operatorname{ALT}\left(\mathrm{U} \mathrm{L}^{-1}\right)$ & \multicolumn{2}{|l|}{$>40$} & $16 / 224(7.1 \%)$ & $11 / 107(10.3 \%)$ & $15 / 117(12.8 \%)$ & 0.6772 \\
\hline $\operatorname{GGT}\left(\mathrm{U} \mathrm{L}^{-1}\right)$ & \multicolumn{2}{|l|}{$>50$} & $33 / 224(14.7 \%)$ & $15 / 107(14.0 \%)$ & $18 / 117(15.4 \%)$ & 0.8513 \\
\hline \multicolumn{7}{|l|}{ Glycaemic control disorders } \\
\hline Hyperglycaemia & \multicolumn{2}{|c|}{$\mathrm{FBS} \geq 6.1 \mathrm{mmol} \mathrm{L}^{-1}$} & $127 / 224(56.7 \%)$ & $51 / 107(47.7 \%)$ & $76 / 117(65.0 \%)$ & 0.0105 \\
\hline Impaired Fasting Glucose & \multicolumn{2}{|c|}{ FBS 6.1 to $6.9 \mathrm{mmol} \mathrm{L}^{-1}$} & $21 / 224(9.4 \%)$ & $8 / 107(7.5 \%)$ & $13 / 117(11.1 \%)$ & 0.3706 \\
\hline Diabetes & \multicolumn{2}{|c|}{$\mathrm{FBS} \geq 7.0 \mathrm{mmol} \mathrm{L}^{-1}$} & $106 / 224(47.3 \%)$ & $43 / 107(40.2 \%)$ & $63 / 117(53.8 \%)$ & 0.0453 \\
\hline
\end{tabular}

\section{Discussion}

This study provided a unique opportunity to look at various characteristics of stroke patients admitted at Komfo Anokye Teaching Hospital in Kumasi. There is good evidence that modification of risk factors will reduce the risk of stroke. This study involving 224 stroke patients, suggests that while there could be differences in some stroke risk factors, between women and men, there are other factors that show no gender differences.

On lifestyle, information gathered on the patients are in Table 1, which shows men reported higher rates of cigarette 
smoking, similar to a study by Husten et al. (2004) and higher alcohol use than women, which is similar to a study by Ofili et al. (1993), which reported that African American men reported higher alcohol and cigarette use than women. In both the Framingham Study and the Nurses' Health Study (Fustinoni and Biller, 2000) cessation of smoking led to a prompt reduction in stroke risk, within 2 to 4 years. This reduction in risk occurred throughout all the age ranges of these studies, and in heavy, as well as moderate smokers.

The study also shows a high prevalence of diabetes; $35.0 \%$ in males and $28.0 \%$ in females. For the overall population of stroke patients, the prevalence of diabetes was found to be $31.7 \%$, which was similar to a study by Hier et al., (1991) who found the prevalence of diabetes to be $33 \%$ in patients with stroke. Baker and Katsuki (1999), found the presence of diabetes in $30 \%$ of Japanese stroke cases. Diabetes is known to predispose to the development of stroke by its contribution to the atherogenesis in the cerebral vessels by the multiple lipid disorders associated with it (Adams et al., 1993).Persons with diabetes have an increased susceptibility to stroke, because of the presence of notably, hypertension, obesity, and abnormal blood lipids (Otvos, 1999). Therefore, it is very important to address the issue of prevention early in life because the precursors of the disease are present long before clinically significant levels of hypertension are recognized (Srinivasan et al., 2003). In this present study, the percentages of hypertension and diabetes between men and women did not differ significantly (p-value of 0.22) (Table1). Hypertension is the single most important modifiable risk factor for stroke. From Table 1, 51.3\% of the studied populations were known hypertensives, which are similar to a study by McDowell et al., (2001) which also reported 51\% of hypertensives. It is clear from the present study that hypertension is similarly associated with both male and female stroke cases. These allude to the major contributory role hypertension plays in the aetiology of stroke.

Hypertension can accelerate atherogenesis in the cerebral vessels, it can mechanically damage these vessels and it can indirectly, through the development of cardiac insufficiency, lead to reduced perfusion in previously narrowed cerebral vessels. It seems that hypertension is indeed one of the main characteristics of the stroke-prone individual. It should be detected early, treated, and controlled. Though the prevalence of diabetes did not show any gender-bias, as pointed out earlier, quite a different pattern emerges when the patients are looked at, based on the disorders of glycaemic control, dependent on some glucose cut-off levels (Table 2). Per the glucose levels, $53.8 \%$ of the males were diabetic, as against $40.2 \%$ of females; the males with hyperglycaemia were as high as $65.0 \%$, as against $47.7 \%$ females. However, the prevalence of impaired fasting glucose showed no difference between males and females.

Whether there will be normal glycaemic control, impaired glucose tolerance, or diabetes, would be determined by various aspects of glucose metabolism that may contribute to risk factors for stroke, including insulin sensitivity, hyperinsulinaemia and increased insulin resistance (the relative inability of insulin to enhance glucose disposal). Hyperinsulinaemia and increased insulin resistance have been shown to be risk factors for stroke among subjects with normal glucose status (Allan et al., (2007).

Another significant finding in this study is that dyslipidaemia, particularly hypertriglyceridaemia, is the most common disorder among the lipid profile test. The rapid nutrition transition in Ghanaians, characterized by a more sedentary lifestyle and an energy-dense higher fat diet may, at least in part, account for the higher prevalence of hypertriglyceridaemia in this Ghanaian study population. This element of nutrition transition is a central contributor of hyperlipidaemia (Yuan et al., 2007).

Among all the dyslipidaemia, the men had the higher percentages except that of increased LDL-C, as shown in Table 2. This is similar to a study by Konishi et al. (2003), who found an increased LDL-C in women. Among the stroke patients, elevated level of serum triglycerides had the highest prevalence of $55.8 \%$, representing more than half of the stroke patients (Table 2).

From this study, it has been observed that elevated blood pressure and elevated fasting glucose are common among stroke patients; this is seen in Table 1, and is similar to a work by McNeill et al. (2006). Therefore, in many of the patients, the stroke could have resulted not only from hypertension alone, but from a combination of hypertension and diabetes, since subjects with hypertension are more likely to develop type 2 diabetes. A study by Gress et al. (2000) had assessed the joint effect of hypertension and type 2 diabetes on stroke risk in the general population. It was found that the risk of stroke attributable to a history of both diabetes and hypertension was substantially greater than for either condition alone, and this has been corroborated by the present study. The proportions of the patients with increased aminotransferases (Table 2) were similar to a study by Perry et al., (2008), in which $23.1 \%$ and $12.8 \%$ of the males had elevated AST and ALT respectively while $15.9 \%$ and $10.3 \%$ of the females had elevated AST and ALT, respectively. Alcohol intake is a main cause of aminotransferase elevation in men but not in women, the relation between aminotransferase and stroke risk can be different by sex (Woo et al., 2002).

\section{Conclusions}

This study identified several risk factors of stroke. There was a high prevalence of both hypertension and hyperglycaemia among the stroke patients. The risk factors identified in the stroke patients were as follows: $63 \%$ had high blood pressure, known hypertensives were 51.3\%, hyperglycaemia $56.7 \%$, diabetes $47.3 \%$, alcoholism $30.4 \%$, hypertriglyceridaemia $55.8 \%$ when the various risk factors were considered.

The highest predominant risk factor observed was hypertension, followed by diabetics and hypertriglyceridaemia. Since the factors responsible for stroke are modifiable, careful consideration should be given 
to the risk factors of stroke in developing countries and appropriate community-based prevention strategies aimed at reducing the frequency of these risk factors should be advocated.

\section{References}

[1] Adams, H.P., Bendixen, B.H.and Kappelle,L.J. (1993) Classification of subtype of acute ischemic stroke: definitions for use in a multicenter clinical trial: TOAST: Trial of Org 10172 in Acute Stroke Treatment. Stroke. 24, 35-41.

[2] Algra, A. (2006) Medium intensity oral anticoagulants versus aspirin after cerebral ischaemia of arterial origin (ESPRIT): a randomised controlled trial. Lancet Neurol. 367, 1665-1673.

[3] Allan, J.D., Mayo, K. and Michel, Y. (2007) Body size values of white and black women. Res Nurs Health.16, 323-333.

[4] Baker A.B.and Katsuki, S. (1999) A study of a Causation and Oriental population. Geriatrics 24, 83-88.

[5] Booth, J. (1999) A short history of blood pressure measurement.Proceedings of the Royal Society of Medicine. 70, 793-799.

[6] Donnan, G.A., Fisher, M., Macleod, M. and Davis, S .M. (2008) Stroke.Lancet. 371, 1612-1623.

[7] Fustinoni, O. and Biller, J. (2000) Ethnicity and stroke: beware of the fallacies. Stroke.31, 1013-1015.

[8] Gress, T.W., Nieto, F.J., Shahar, E., Wofford, M.R.and Brancati, F.L. (2000) Hypertension and antihypertensive therapy as risk factors for type 2 diabetes mellitus. Atherosclerosis Risk in Communities Study. $N$ Engl $J$ Med. 342, 905- 912.

[9] Grundy, S.M., Brewer, H.B., Cleeman, J.I., Smith, S.C. and Lenfant, D. (2004) Definition of metabolic syndrome: report of the National, Heart, Lung, and Blood Institute/American Heart Association conference on scientific issues related to definition.Circulation. 109, 433-438.

[10] Hier, D.B. Foulkes, M.A., Swiontoniowski, M., Sacco R.L., Gorelick, P.B., Mohr, J.P., Price, T.R. and Wolf
P.A.(1991)Stroke recurrence within 2 years after ischemic infarction.Stroke. 22, 155-161.

[11] Husten, C.G., Shelton, D.M, .Chrismon, J.H., Lin, Y.C., Mowery, P. and Powell, F.A. (2004) Cigarette smoking and smoking cessation among older adults.Tob Control.6, 175-180.

[12] Konishi, M., Iso, H., Komachi, J., Shimamoto, T., Jacobs, D.R. and Terao, A. (2003) Associations of serum total cholesterol, different types of stroke, and stenosis distribution of cerebral arteries: The Akita Pathology Study. Stroke. 24, 954-964.

[13] McDowell, F., Potes, J. and Groch, S. (2001) The natural history of internal carotid and vertebral-basilar artery occlusion. Neurology. 11, 153-157.

[14] McNeill, A.M., Rosamond, W.D. and Girman C.J. (2006) The Metabolic Syndrome and 11-Year Risk of Incident Cardiovascular Disease in the Atherosclerosis Risk in Communities Study. Diabetes Care.28, 385-390.

[15] Ofili, E.O., Kern, M.J. and Labovitz, A.J., (1993) Analysis of coronary blood flow velocity dynamics in angiographically normal and stenosed arteries before and after endolumen enlargement by angioplasty. J Am Coll Cardiol. 21, 308-311.

[16] Otvos, J. (1999) Measurement of triglyceride-rich lipoproteins by nuclear magnetic resonance spectroscopy. Clin Cardiol. 22, I21-172.

[17] Perry, I.J., Wannamethee, S.G. and Shaper, A.G. (1998) Prospective study of serum $\gamma$-glutamyltransferase and risk of NIDDM.Diabetes Care. 211, 732-737.

[18] Srinivasan, S.R., Myers, L. and Berenson, G.S. (2003) Predictability of childhood adiposity and insulin for developing insulin resistance syndrome (syndrome $\mathrm{X}$ ) in young adulthood. The Bogalusa Heart Study.Diabetes.51, 204-209.

[19] Woo, J., Lau, E. and Kay, R. (2002) Elderly subjects aged 70 years and above have different risk factors for ischemic and hemorrhagic strokes compared to younger subjects. $J \mathrm{Am}$ Geriatric Soc. 40, 124-129.

[20] Yuan, G., Al-Shali, K.Z. and Hegele, R.A. (2007) Hypertriglyceridemia: its etiology, effects and treatment. CMAJ.176,113-120. 